\title{
¿Qué Tipo de Docentes Tutorizan las Prácticas de los Futuros Maestros de Primaria?
}

\author{
What Kind of Teachers Guide the Future Primary School \\ Teacher's Internship?
}

\author{
Ernesto Colomo * \\ Vicente Gabarda \\ Universidad Internacional de Valencia, España
}

\begin{abstract}
Durante las prácticas curriculares, los tutores de los centros se convierten en ejemplos para el desarrollo profesional de los estudiantes. En este sentido, el fin de esta investigación es identificar los arquetipos de docentes que tutorizan a los futuros maestros de primaria, a partir de la propuesta de Esteban y Mellen (2016). La muestra está compuesta por 230 estudiantes matriculados en las cuatro asignaturas de Prácticas Escolares del Grado en Educación Primaria de la Universidad Internacional de Valencia durante el curso 2017/2018. Partiendo de una perspectiva cualitativa de carácter descriptivo-interpretativo, se han analizado las características de los tutores de los centros a partir del análisis de contenido de los diarios de prácticas. Los resultados muestran que el arquetipo de docente más presente entre los tutores es el del maestro auténtico y personal, destacando una actitud de cuidado hacia las relaciones profesionales y personales con el alumnado. También se han recogido aspectos como el dominio de la materia en la tipología del maestro transmisor de historias o la innovación educativa en el maestro motivador para el aprendizaje. Las conclusiones inciden en la importancia de la forma de ser y ejercer la labor docente por el tutor del centro debido a su influencia en la construcción de la identidad profesional docente de los estudiantes.
\end{abstract}

Descriptores: Formación de docentes de primaria; Estudiante de prácticas; Competencias del docente; Identidad; Profesor de primaria.

During the internship, the school's tutors become examples for the professional development of the students. In this sense, the purpose of this research is to identify the archetypes of teachers who guide to the future primary teachers, based on the proposal of Esteban y Mellen (2016). The sample is composed by 230 students enrolled in any of the four internship subjects of the Primary Education Degree, in the 2017-2018 course. Based on a qualitative descriptive-interpretative perspective, the characteristics of the tutors in the workplaces have been analysed, through the content analysis of the students' reports. The results show that the most frequent archetype is the personal and authentic teacher, highlighting a caring attitude towards the professional and personal relationships with the students. Aspects like the content domain of the teacher transmitter of stories or the educational innovation of the teacher motivator of learning have also been collected. The conclusions suggest the importance of the way of being and exercising the educational processes from the tutors due to their influence in the students' teacher professional identity construction.

Keywords: Primary teacher training; Trainees; Teacher qualifications; Identity; Primary school teachers.

*Contacto: ecolomomagana@gmail.com

Recibido: $\quad 19$ de marzo 2019

$1^{\text {a }}$ Evaluación: 30 de abril 2019

ISSN: $1696-4713$

$2^{\text {a }}$ Evaluación: 7 de mayo 2019

www.rinace.net/reice/

Aceptado: 15 de mayo 2019

revistas.uam.es/reice 


\section{Introducción}

Basta un solo profesor -jtan solo uno!para salvarnos de nosotros mismos.

(Pennac, 2008, p. 219)

Las prácticas curriculares, ligadas a la formación inicial, son un periodo clave de aprendizaje real sobre el ejercicio y la labor docente, así como para la construcción identitaria de los futuros maestros de primaria. En este sentido, los tutores de los centros se convierten en ejemplos y guías, puesto que su forma de actuar durante el proceso de enseñanza-aprendizaje influye en la adquisición y desarrollo de competencias, actitudes y valores por parte de los estudiantes de magisterio. El fin es identificar los arquetipos de docentes que tutorizan a estos estudiantes durante su periodo de prácticas curriculares. Para ello, se realizará un análisis pormenorizado de los diarios de prácticas utilizando como categorías informativas las tipologías de maestros defendidas pos Esteban y Mellen (2016).

\section{Revisión de la literatura}

En la formación de cualquier profesión, se necesita un componente práctico para poder aplicar los conocimientos adquiridos en escenarios reales de intervención (Valle y Manso, 2018). En este sentido, las prácticas escolares son la primera experiencia del futuro docente en un aula tras haber tomado la decisión de dedicar su vida a la docencia, de ahí que sea considerado un elemento fundamental en el proceso de formación inicial por diferentes autores (Cid, Pérez y Sarmiento, 2011; González, 2015; Zabalza, 2011). Se trata del comienzo del proceso de socialización laboral (Bodas, González y Martín, 2016), permitiendo al estudiante trasladar los conocimientos teóricos adquiridos durante la formación inicial al contexto real del aula (García, 2017), donde se suceden múltiples experiencias que favorecerán el aprendizaje de la profesión docente (García, González y Martín, 2016; González y Medina, 2017; Zabalza, 2016). Estamos, por tanto, ante un periodo formativo clave para los estudiantes de magisterio por su utilidad (Ries, Yanes y Ballesteros, 2014) y que es muy valorado (Manso y Martín, 2014; Melgarejo, Pantoja y Latorre, 2014), ya que las prácticas escolares les permiten vivenciar tareas profesionales (Tejada, Carvalho y Ruiz, 2017). A nivel científico, su importancia ha generado una amplia y variada producción, situando el foco en diferentes aspectos como el desarrollo de las competencias docentes del alumnado, la importancia de la figura del tutor del centro (Palomares y Alarcón, 2018) o la construcción de la identidad profesional docente (Tejada y Ruiz, 2013). Serán estos dos últimos aspectos donde situaremos el foco de este estudio. El motivo es que las prácticas sitúan a los futuros docentes ante un nuevo escenario, donde los recuerdos de las experiencias e interacciones que tenían de su época de estudiantes chocan con un análisis crítico de la labor docente que ejerce su tutor de prácticas. Se trata, por tanto, de cambiar progresivamente el foco y la perspectiva respecto al proceso de enseñanza-aprendizaje en función de cómo se responde a la pregunta ¿quiénes queremos ser como maestros? Para responder a esta cuestión, debemos incidir en que la profesión docente no se reduce a dominar los contenidos, conocer diferentes metodologías y estrategias de aprendizaje o conectar psicosocialmente con el alumnado. Ser maestro es más que la suma de nuestras competencias, conocimientos, valores y actitudes, es una forma de vida. Esta transformación que debe producirse respecto al rol, pasando de la visión de estudiante a la de futuro maestro, es crucial para el proceso de (re)construcción 
de la identidad profesional docente, pasando a comentar los aspectos claves de la misma a continuación.

La identidad profesional docente (IPD) se origina como fruto de las experiencias e interacciones en el contexto escolar, en la que influyen las vivencias durante la época de alumno, la formación inicial-continua como docente y la propia praxis del ejercicio como maestro, comprendiendo así diferentes escenarios (Granados, Tapia y Fernández, 2017; Sarceda, 2017) que dan lugar a identidades únicas. Se trata de un ámbito cada vez más estudiado (Cantón y Tardif, 2018; Hong, Greene y Lowery, 2017). Está compuesta por una serie de aspectos individuales (elementos personales, como los valores, y cognitivos, como el conocimiento sobre la asignatura) y sociales (cuestiones culturales, políticas, sociales, económicas, etc.) y con un carácter dinámico, motivo que favorece la adaptación pedagógica del maestro (Santamaría, Torrego y Ortiz, 2018). Por tanto, los procesos de reconstrucción identitaria docente son permanentes, debido a que están ligados a todo cambio que se produce en el binomio razón-acción derivado de su intervención en el contexto escolar. En este sentido, es preciso subrayar que el desarrollo de la IPD no está sujeto al número de vivencias o tiempo de experiencia en el aula, si no al significado que se le atribuyen a las mismas y la consecuente adquisición de conocimientos que se deriva de analizarlas de forma reflexiva. Por lo tanto, es la reflexión sobre la praxis educativa (propia o ajena, siendo también la observación crítica un factor enriquecedor para el desarrollo de las competencias vinculadas al ejercicio docente) el medio para evolucionar y perfeccionarnos como maestros. Los procesos de autorreflexión sobre la identidad docente permiten al estudiante de magisterio conocerse mejor así mismo a nivel profesional (Ramírez y de la Herrán, 2012), influyendo en dos aspectos claves: por un lado, es capaz de analizar críticamente las diferentes intervenciones educativas que se producen en el aula, apostando por implementar aquellas que resultan exitosas para el proceso de enseñanza-aprendizaje de los discentes; y por otro lado, consigue detectar aquellas competencias, destrezas o actitudes profesionales y personales que debe seguir reforzando y consolidando para crecer y evolucionar como docente. En este sentido, a través de las prácticas escolares, los futuros docentes podrán auto-chequear diferentes aspectos (Zabalza, 2016) como el nivel de adquisición de las diferentes competencias docentes o su carácter como maestro (Esteban, 2018). En este sentido, tener una buena guía y ayuda durante la experiencia formativa en prácticas puede facilitar tanto el proceso formativo y madurativo en cuanto a nuestra IPD (Romera, 2011) como el cambio y definición profesional, de ahí la importancia que adquiere la figura del tutor del centro.

Antes de profundizar en el papel de esta figura, es necesario subrayar que el alumno que debe realizar las prácticas ya tiene una idea preconcebida de la realidad del aula y de cómo ejercer la función docente. Investigaciones como la de Mellado, Ruiz, Bermejo y Jiménez (2006), inciden en la propensión que tienen los estudiantes de magisterio en replicar la forma de enseñar en la que ellos fueron educados. De esta manera, cuando se incorporan a los centros de prácticas, su creencia sobre las competencias, características o requerimientos que debe tener la figura docente estará determinada, en cierto modo, por las interacciones y experiencias que acontecieron con los distintos profesores que formaron parte de su etapa educativa (Bodas et al., 2016). No obstante, la primera experiencia con un docente tras elegir esta profesión también debe valorarse, como hemos comentado, debido al gran poder de influencia que las vivencias junto al tutor y su ejemplo tienen tanto en la construcción de la IPD (López, Torrego y Vallés, 2015; Martínez y Raposo, 2011) como en el éxito o fracaso de dicho periodo formativo. De esta manera, 
siguiendo a González y Fuentes (2011), encontramos que el futuro docente "se verá influenciado tanto por su historia personal y sus experiencias previas como por las características del contexto de prácticas en el que se desarrolla y con el que interacciona para construir significados e interpretaciones idiosincráticas, distintas y únicas” (p. 62). Debemos partir de la consideración de que el tutor posee un background respecto al hecho educativo que le permite actuar y tomar decisiones con seguridad, mientras que el estudiante en prácticas adolece de esa experiencia al no haberse enfrentado a un contexto real de forma autónoma (Leguizamon, 2014). Así, el tutor se transforma en el referente del futuro docente al acompañarle, guiarle y orientarle en su intervención en el aula, promoviendo de forma progresiva su autonomía y un rol activo como futuro docente (Egido y López, 2016; Tejada y Ruiz, 2013) en un contexto de experimentación real. Para ello, es clave que se construya una relación de confianza y seguridad entre ambos agentes, con el fin de que el estudiante en prácticas se sienta libre de consultar y opinar de forma crítica sobre cualquier cuestión de carácter organizativo, legislativo o pedagógico, mostrando el tutor una actitud disponible y de ayuda para resolver las dudas y necesidades que le pueda plantear (Colén y Castro, 2017). También le aporta un equilibrio entre conocimientos teóricos y prácticos (González, Martín y Bodas, 2017), favoreciendo un cuestionamiento de las creencias pedagógicas que tiene el estudiante mediante un proceso de autoanálisis a raíz de las intervenciones en el contexto escolar (Sepúlveda et al., 2017), facilitando así la consecución de aprendizajes reflexivos que beneficien a su desarrollo profesional (Cid et al., 2011; Ralph y Walker, 2014). Es preciso también considerar que los tutores representan diferentes arquetipos de docentes. En este sentido, los arquetipos hacen referencia a constructos y patrones generados a nivel cultural (Jung, 1992, 1994) que engloban diferentes características. Estos dan lugar a diferentes modelos o prototipos docentes a partir de los rasgos profesionales y personales que los definen. En función del arquetipo que los docentes representen, aportarán una serie de enseñanzas, valores, actitudes, etcétera, que influirán en el proceso de (re)construcción identitaria de los futuros maestros que se encuentran en prácticas. Como fruto de aunar diferentes propuestas de índole internacional y nacional (Beauchamp y Thomas, 2009; Jordán, 2003; Toterhi y Hancock, 2007) sobre las características y competencias que debe tener un buen docente, Esteban y Mellen (2016) plantean un modelo en el que identifican tres tipologías de maestros en función de los rasgos que las definen.

Maestro auténtico y personal. Se caracteriza por su empatía y por los lazos afectivos que genera con los discentes (Day, 2009), a nivel académico y personal, en una relación de desarrollo y perfeccionamiento conjunto (Scott, 2000). Muestra así una alta competencia emocional (Day, 2011), lo que le permite ayudar al alumnado en el control y regulación de sus emociones. Confía en su influencia positiva para el desarrollo y bienestar del educando (Jordán, 2013), convirtiéndose en ejemplos a los que imitar (Steiner y Ladjali, 2005). Trata, por tanto, de implementar procesos educativos personalizados que atiendan las necesidades y particularidades del alumnado, sin descuidar su propia evolución mediante el ejercicio del magisterio, trabajando aspectos como el carácter o la moral (Matsuba, Murzyn y Hart, 2011). Su vocación hacia el ejercicio docente es un pilar clave en su construcción identitaria (Esteve, 2009) y en su entrega para con el discente. Se trata, en definitiva, de docentes implicados en el desarrollo integral tanto de su alumnado como del suyo propio.

Maestro transmisor de historias. Se caracteriza por un dominio profundo de su asignatura y por aunar amor, pasión e inquietud hacia su disciplina (Fried, 2001; Muñoz, 2010), 
favoreciendo así el enriquecimiento de sus lecciones mediante su amplia formación cultural (López, 2016; Recalcati, 2016). Su nivel de conocimiento le permite establecer vínculos entre los contenidos de su asignatura y lo que acontece en la realidad (Oakeshott, 2009), dando lugar a un proceso de aprendizaje más holístico debido a su aplicabilidad. Es la forma apasionada de transmitir en sus clases lo que despierta el interés y la motivación del alumnado hacia la misma (Buxarrais, Vilafranca y Bujons, 2016; Ordine, 2013). Estamos ante un maestro entregado tanto a su asignatura como a favorecer el proceso de aprendizaje de la misma.

Maestro motivador para el aprendizaje. Domina los procesos didácticos-pedagógicos, diferentes metodologías, recursos y estrategias didácticas, lo que le permite desarrollar procesos de enseñanza-aprendizaje positivos (Applegate, 2010; Martín, Conde y Mayor, 2014), lo que denota un alto desarrollo de la competencia pedagógica. Apuesta por dar el protagonismo al alumnado en procesos formativos mediados por la creatividad, la innovación y la responsabilidad con la mejora continua (Centeno et al., 2017; Quevedo y Arruti, 2018), consiguiendo así despertar en los discentes el deseo de aprender y la motivación sin que ello le haga perder el control ni la responsabilidad sobre la evolución académica de los mismos (Mojarro, Rodrigo y Etchegaray, 2015).

En este sentido, el análisis y la reflexión sobre el periodo de prácticas escolares por parte de los estudiantes de magisterio permitirá identificar la tipología de docente que les han tutorizado durante dicho proceso formativo, pudiendo presentar características de los diferentes arquetipos al no ser excluyentes entre sí. Dicha información la obtendremos de los diarios de prácticas que realiza el alumnado.

Y es que en los diarios se narran las experiencias vividas, de forma pormenorizada, por los estudiantes de magisterio desde su perspectiva personal, ensalzando aquellos acontecimientos que por su novedad, relevancia o curiosidad les parecen importantes y consideran que deben ser registrados. De esta manera, mediante un proceso de reflexión crítica (Aguilar, 2017; Lopes y Blázquez, 2012), dotan a las vivencias del aula de significado a partir de los conocimientos teóricos que adquieren durante la formación inicial, aprendiendo así de las diferentes experiencias que les ofrece la realidad del contex to escolar. Así, los diarios permiten conocer "qué está viviendo el alumnado en sus prácticas, cómo lo están interpretando, qué posibles creencias hay subyacentes y cómo están tratando de resolver las situaciones cotidianas de la práctica educativa con las que se enfrentan cada día” (Sepúlveda et al., 2017, p. 97). Esto les permite desarrollar tanto su pensamiento reflexivo como su identidad docente (López et al., 2015), favoreciendo un conocimiento más profundo de sí mismos respecto al aspecto profesional (Leguizamon, 2014). Este hecho da lugar a que cada diario sea único, pues, a la ya de por sí variada experiencia derivada de cuestiones contextuales como la idiosincrasia de cada centro o de las características diferenciales de los estudiantes, hay que añadirle la singular relación que se produce entre la interpretación subjetiva y personal de la praxis en el aula de cada futuro docente con el nivel de conocimiento pedagógico que posea. De esta manera, la IPD queda reflejada en los diarios al ir narrando en primera persona la intervención en el periodo de prácticas (García, 2017), permitiéndonos conocer tanto el modo en que se ha producido el aprendizaje del alumno como los puntos fuertes y débiles del mismo. El estudio de los diarios de prácticas se ha utilizado, entre otros aspectos, para analizar el desarrollo de las competencias profesionales (Medina et al., 2013) y como instrumento de reflexión y relación entre teoría y práctica (Corral y Cacheiro, 2016; González, 2015). En nuestro caso, profundizaremos en los diarios de los estudiantes de magisterio para conocer 
qué rasgos caracterizaban a sus tutores de prácticas y la influencia que los diferentes arquetipos presentes tienen en la construcción de la identidad profesional docente del alumnado.

El objetivo general de esta investigación es identificar los arquetipos de docentes que tutorizan a los estudiantes del Grado en Educación Primaria de la Universidad Internacional de Valencia durante su periodo de prácticas curriculares en los centros educativos.

Entre los objetivos específicos encontramos los siguientes:

- Analizar la frecuencia de cada uno de los diferentes arquetipos y sus respectivos rasgos definitorios en los centros de prácticas.

- Identificar diferencias entre los arquetipos de tutores presentes en los centros, según la percepción de los estudiantes, en función del periodo de prácticas cursado.

- Registrar evidencias sobre los rasgos definitorios de los tutores atribuidos a cada arquetipo según la visión de los estudiantes en prácticas.

\section{Método}

\section{Enfoque metodológico}

Desde un punto de vista metodológico, se presenta un estudio mixto de carácter descriptivo-interpretativo sobre los principales arquetipos docentes asociados a los tutores de prácticas curriculares de los centros educativos, fruto del análisis sobre las experiencias narradas por estudiantes de magisterio de la etapa de primaria en sus diarios. Utilizaremos como método el análisis de contenido (Bardín, 1986), una técnica que une la lectura e interpretación del material objeto de estudio y permite examinar las ideas expresadas en los diarios, centrando la atención en el significado otorgado por el alumnado a las evidencias, las cuales se cuantificarán e interpretarán. Se trata de un método que ha sido implementado en diferentes estudios (Cabero et al., 2016; Fontal et al., 2017; Travé, Estepa y Delval, 2017), y que nos permitirá conocer las experiencias y reflexiones registradas en los diarios que están relacionadas con los arquetipos docentes que representan los tutores de prácticas del centro.

\section{Muestra}

La muestra objeto de estudio estará conformada por los estudiantes que cursan cualquiera de los cuatro periodos de prácticas curriculares del Grado en Educación Primaria de la Universidad Internacional de Valencia, matriculados en el curso académico 2017/2018. La muestra, seleccionada de forma intencionada (no probabilística), ha quedado finalmente compuesta por 230 estudiantes $(\mathrm{N}=230)$, donde el $81,73 \%$ son mujeres y el $18,27 \%$ hombres. La distribución del alumnado, en función del periodo de prácticas en que se hallaba matriculado, ha sido la siguiente: 28 estudiantes de Prácticas I (12,18\%), 57 de Prácticas II (24,782\%), 80 de Prácticas III (34,782\%) y 65 de Prácticas IV (28,26\%). En este sentido, contamos con una muestra semejante a otros estudios que también han analizados diarios de prácticas escolares, como el de Cebrián, Pérez y Cebrián (2017) con una muestra total de 192 diarios, y siendo muy superior a otros como el de González, Martín y Bodas (2017) donde se analizaron 12 diarios. 


\section{Instrumento}

Para la recogida de la información, partiremos de los diarios de prácticas realizados por el alumnado. Dicho registro de las acciones y tareas, que pueden recopilarse atendiendo a diferentes aspectos como la temporalidad o las materias, se realiza durante el transcurso de las prácticas para recoger las experiencias vividas. El análisis de los diarios de prácticas se hará desde la perspectiva nomotética (Colomo y Domínguez, 2015) con el fin de poder entender las vivencias registradas por los estudiantes de magisterio en prácticas, empleando como método para su exploración el análisis de contenido.

\section{Trabajo de campo}

Compilada toda la documentación, se inicia el proceso de análisis de contenido de los diarios, siguiendo las fases estipuladas por diferentes autores (Colomo y de Oña, 2014; Sauquillo, Ros y Bellver, 2008): a) pre-análisis, donde se prepara y organiza el material. Para ello, se realizó una lectura inicial exploratoria sobre el contenido de los diarios, para acotar todas aquellas experiencias y vivencias de los estudiantes del grado en educación primaria relacionadas con la figura del tutor de prácticas. Tras ello, en una segunda lectura, se descartaron todas las evidencias que no tuvieran relación con la praxis docente de los tutores de práctica, con el fin de delimitar la información para el análisis; b) establecimiento del sistema categorial, donde definiremos y elaboraremos el instrumento en el que se organizará la información de cara al análisis. El sistema utilizado en esta investigación partirá de los arquetipos docentes propuestos por Esteban y Mellen (2016), incluyendo tres subcategorías en cada arquetipo que permiten una comprensión más significativa del constructo de dicho modelo docente. A partir de estos rasgos definitorios de cada arquetipo, se clasificarán las evidencias para su posterior cuantificación y análisis (figura 1); c) codificación, donde transformaremos las evidencias en unidades más simples para trabajar posteriormente en su cuantificación y descripción; d) interpretación, tanto de corte cuantitativo como cualitativo, donde se dotará de significado a la información extraída de los diarios respecto a las tipologías de docente que representan los tutores de prácticas de los centros y los rasgos definitorios de estas.

\section{Análisis de datos}

Tras aplicar la fase de pre-análisis y la creación del sistema categorial, el análisis de datos se centrará en las fases de codificación e interpretación. Durante la codificación, las unidades textuales que componían las evidencias extraídas de los diarios a partir del sistema de categorías, fueron inicialmente simplificadas (codificadas alfanuméricamente) para asociarlas, por su significado, a los diferentes arquetipos y rasgos definitorios. En la interpretación, primero se desarrolló un proceso descriptivo (a nivel cuantitativo) a partir del registro de evidencias en el sistema de categorías; tras ello, se analizan ejemplos ilustrativos de los diferentes rasgos definitorios de cada uno de los arquetipos trabajados, considerando para dicha interpretación el periodo de prácticas del estudiante y la significatividad que cada rasgo tiene dentro del arquetipo al que pertenece, considerando para ello nuevamente las frecuencias registradas. 


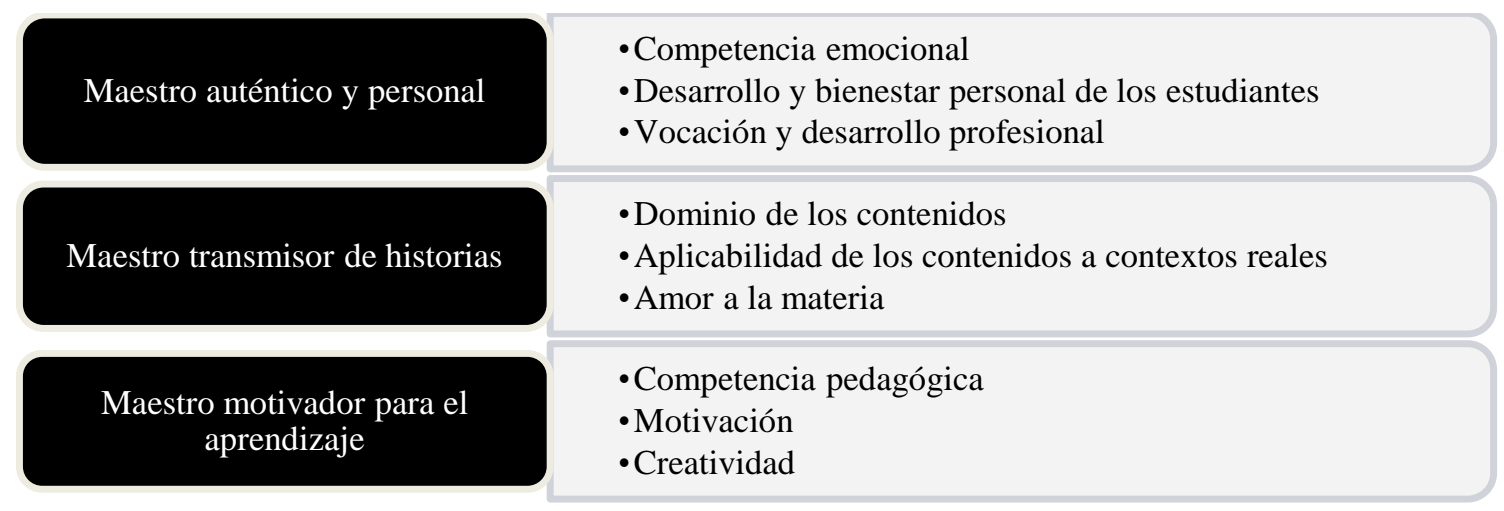

Figura 1. Sistema de categorías

Fuente: Elaboración propia.

\section{Resultados}

Los resultados son fruto del trabajo de observación e interpretación sobre las experiencias y vivencias narradas en los diarios de prácticas escolares analizados. Seguidamente, se presentan los resultados de corte cuantitativo, con el registro de frecuencias de las evidencias, y los de ámbito cualitativo, con la interpretación de las experiencias a partir de las características de las diferentes tipologías de maestros.

\subsection{Enumeración y registro de las evidencias}

Es importante subrayar que el número de evidencias no es coincidente con el total de diarios analizados, ya que los estudiantes de magisterio en prácticas pueden identificar características de los arquetipos en su propio tutor, ya que no son excluyentes entre sí. A continuación, recogemos las frecuencias obtenidas (cuadro 1) respecto a las diferentes tipologías de maestro. En este sentido, se ha asociado a cada arquetipo una serie de rasgos que permiten definir los comportamientos, competencias y actitudes que quedan englobados bajo estos constructos de modelos/tipologías de docentes.

Los datos reflejan que la tipología de maestro más presente entre los tutores, a tenor de la percepción del alumnado en prácticas respecto a las características de estos como docentes, es el arquetipo del maestro auténtico y personal, registrando 212 evidencias que representan el 72,36\% del total. En este sentido, el maestro motivador para el aprendizaje es el siguiente más frecuente, con un total de 59 evidencias (20,14\%), terminando con el maestro transmisor de historias, que ha registrado 22 evidencias $(7,50 \%)$. En lo que respecta al maestro auténtico y personal, el rasgo definitorio más frecuente es la vocación y desarrollo profesional (43,90\%), seguido del desarrollo y bienestar personal de los estudiantes $(31,60 \%)$, encontrándose en último lugar la competencia emocional (24,50\%). En cuanto al maestro transmisor de historias, el rasgo de aplicabilidad de los contenidos a contextos reales representa el $50 \%$ de las evidencias registradas, mientras que el dominio de los contenidos supone el $31,80 \%$ y el amor por la materia el 18,20\%. Por último, en el maestro motivador para el aprendizaje, el rasgo definitorio más frecuente según las evidencias registradas es la motivación (50,80\%), seguida de la competencia pedagógica $(33,90 \%)$ y la creatividad $(15,30 \%)$. 
Cuadro 1. Frecuencias registradas sobre los diferentes tipos de maestros y sus rasgos definitorios

\begin{tabular}{llcc}
\hline \multicolumn{1}{c}{ TiPO DE MAESTRO } & \multicolumn{1}{c}{ RASGOS DEFINITORIOS } & FRECUENCIA & TOTAL \\
\hline \multirow{4}{*}{ Auténtico y personal } & Competencia emocional & 52 & \\
& $\begin{array}{l}\text { Desarrollo y bienestar personal de los } \\
\text { estudiantes } \\
\text { Vocación y desarrollo profesional }\end{array}$ & 67 & 212 \\
& Dominio de los contenidos & 93 & \\
\hline \multirow{2}{*}{ Transmisor de historias } & Aplicabilidad de los contenidos a & 7 & 22 \\
& contextos reales & 11 & \\
\hline \multirow{2}{*}{$\begin{array}{c}\text { Amor a la materia } \\
\text { aprendizaje }\end{array}$} & Competencia pedagógica & 20 & \\
& Motivación & 30 & 59 \\
\hline
\end{tabular}

Fuente: Elaboración propia.

Centrándonos en la distribución por periodos de prácticas, el maestro auténtico y personal se mantiene siempre como el más frecuente en todos los periodos, siendo la vocación y el desarrollo profesional el rasgo definitorio que registra un mayor número de evidencias en los mismos. De esta forma, son las características vinculadas al afecto, la empatía y la preocupación por el alumnado las que más impactan en los futuros docentes durante su periodo de prácticas, recopilándose un mayor número en los diarios. En prácticas I, el porcentaje de evidencias recopiladas es de un $82,2 \%$. En este mismo periodo, el maestro motivador para el aprendizaje registra un 11,1\%, mientras que el transmisor de historias obtiene un $6,7 \%$. En el segundo periodo de prácticas, el maestro auténtico y personal registra un $75,0 \%$ de las evidencias, seguido del motivador para el aprendizaje $(15,3 \%)$ y del transmisor de historias $(9,7 \%)$. En el tercer periodo de prácticas, destaca nuevamente el maestro auténtico y personal (72,7\%), donde el motivador para el aprendizaje obtiene el $19,2 \%$ de las evidencias y el transmisor de historias un 8,1\%. Finalmente, en prácticas IV, el docente auténtico y personal registra un $63,6 \%$, mientras que el motivador para el aprendizaje alcanza el $31,2 \%$ y el transmisor de historias un 5,2\%. Como se puede observar, la distribución de los arquetipos ha sido constante en los diferentes periodos, ocupando el maestro motivador para el aprendizaje el segundo lugar y el transmisor de historias el tercero. Por último, es destacable como los estudiantes en prácticas van aumentando su valoración respecto al arquetipo del maestro motivador para el aprendizaje, tomando un mayor protagonismo las aptitudes pedagógicas-didácticas de los tutores e incrementando su relevancia para la conformación de la identidad docente según avanzan los periodos de prácticas.

\subsection{Análisis e interpretación de las evidencias}

El análisis interpretativo se centrará en los rasgos definitorios de los tutores que evidencian los estudiantes en prácticas, las cuales se asocian a los diferentes arquetipos de maestros. Partimos, de esta manera, de la convicción de que las actitudes, destrezas y competencias que los tutores pongan en juego serán las que más influyan en los futuros docentes respecto a la construcción de su IPD. Abordaremos cada tipología docente, a continuación, de forma pormenorizada. 
Cuadro 2. Frecuencias registradas sobre los diferentes tipos de maestros y sus rasgos definitorios en cada periodo de prácticas

\begin{tabular}{|c|c|c|c|c|c|}
\hline $\begin{array}{c}\text { TIPO DE } \\
\text { MAESTRO }\end{array}$ & $\begin{array}{c}\text { RASGOS } \\
\text { DEFINITORIOS }\end{array}$ & $\begin{array}{c}\text { PrÁCTICAS } \\
\text { I } \\
\end{array}$ & $\begin{array}{c}\text { Prácticas } \\
\text { II }\end{array}$ & $\begin{array}{c}\text { PRÁCTICAS } \\
\text { III }\end{array}$ & $\begin{array}{c}\text { Prácticas } \\
\text { IV }\end{array}$ \\
\hline \multirow{4}{*}{$\begin{array}{c}\text { Auténtico y } \\
\text { personal }\end{array}$} & $\begin{array}{c}\text { Competencia } \\
\text { emocional }\end{array}$ & 7 & 14 & 18 & 13 \\
\hline & $\begin{array}{l}\text { Desarrollo y } \\
\text { bienestar personal } \\
\text { de los estudiantes }\end{array}$ & 11 & 18 & 23 & 15 \\
\hline & $\begin{array}{l}\text { Vocación y } \\
\text { desarrollo profes. }\end{array}$ & 19 & 22 & 31 & 21 \\
\hline & Total & 37 & 54 & 72 & 49 \\
\hline \multirow{4}{*}{$\begin{array}{l}\text { Transmisor } \\
\text { de historias }\end{array}$} & $\begin{array}{l}\text { Dominio de los } \\
\text { contenidos }\end{array}$ & 2 & 3 & 2 & O \\
\hline & $\begin{array}{l}\text { Aplicabilidad de los } \\
\text { contenidos a } \\
\text { contextos reales }\end{array}$ & $\mathrm{O}$ & 2 & 5 & 4 \\
\hline & Amor a la materia & 1 & 2 & 1 & O \\
\hline & Total & 3 & 7 & 8 & 4 \\
\hline \multirow{4}{*}{$\begin{array}{l}\text { Motivador } \\
\text { para el } \\
\text { aprendizaje }\end{array}$} & $\begin{array}{r}\text { Competencia } \\
\text { pedagógica }\end{array}$ & $\mathrm{O}$ & 3 & 8 & 9 \\
\hline & Motivación & 5 & 6 & 8 & 11 \\
\hline & Creatividad & $\mathrm{O}$ & 2 & 3 & 4 \\
\hline & Total & 5 & 11 & 19 & 24 \\
\hline
\end{tabular}

Fuente: Elaboración propia.

\subsubsection{Maestro auténtico y personal}

El ámbito emocional es una realidad que cobra cada vez mayor importancia en el contexto escolar para el desarrollo integral del alumnado. En este sentido, es clave abordar los aspectos emocionales, éticos y sociales durante la formación inicial (Day, 2017; Hargreaves, 2005), debiendo trabajarse tanto a nivel teórico como práctico, donde el ejemplo del tutor del centro de prácticas resulta enriquecedor. Así, encontramos tutores que priorizan cómo se sienten sus estudiantes y el factor humano sobre otras cuestiones de índole académica. De este modo, la competencia emocional se convierte en un rasgo clave en esta tipología docente, adquiriendo mayor relevancia en las evidencias a partir del segundo periodo de prácticas, debido a que el alumnado posee un mayor nivel de desarrollo formativo.

\footnotetext{
Los conoce muy bien y puede incidir en aquellos aspectos emocionales que considera más necesarios en todos ellos, tanto a nivel individual como a nivel grupal. Ojalá estos detalles fuesen tenidos en cuenta por la mayoría de maestros y maestras, ya que nuestro objetivo no es darles solo una formación académica, sino contribuir a su desarrollo integral como personas. (S.119 - Prácticas III)

Me ha encantado de mi tutora de prácticas, no solo la profesionalidad que demuestra, sino también la humanidad con respecto a los alumnos y alumnas, siempre priorizando su bienestar físico y psicológico sobre los conocimientos que han de adquirir. (S.55 - Prácticas II)
}

Nos situamos ante un modelo de docente que comprende la importancia de ayudar en la regulación emocional de su alumnado (Aguaded y Pantoja, 2015; Day, 2011), y que, apuesta por humanizar el proceso educativo, incluyendo tanto la formación como la propia interacción y vida en la realidad del aula (Frankl, 2013). El cuidado respecto a las 
interrelaciones entre maestro y alumnos, la preocupación por el bienestar personal de estos y una actitud empática y de cariño hacia los discentes genera un clima socio-afectivo positivo para el desarrollo de los procesos de enseñanza-aprendizaje, siendo el segundo rasgo que más evidencias ha registrado dentro de este arquetipo. Destacan evidencias de los dos primeros periodos de prácticas, donde la relación profesor-alumno resulta clave para los estudiantes en sus primeras experiencias como docentes en prácticas, modelando a partir del ejemplo del tutor del centro la forma en la que establecerán este tipo de relaciones.

\begin{abstract}
La relación que se puede observar va más allá del interés por dar bien una clase o por enseñar unos conceptos a los alumnos, existe una relación personal que hace que los alumnos y el profesor formen un equipo. (S.73 - Prácticas II)

Este profesor me llamó especialmente la atención ya que, tenía una forma muy particular de tratar a sus alumnos. Los trataba con muchísimo cariño, casi como si fuera un amigo y era capaz de hacerse respetar y poner a la clase entera "firme" sin tener ni siquiera que levantar la voz. Creo que es un profesor muy empático con un gran respeto y mucha comprensión hacia sus alumnos y la verdad es que me dejó muy sorprendida la forma en la que organizaba sus clases. (S. 10 - Prácticas I)
\end{abstract}

Los tutores que se perciben con el arquetipo de maestro auténtico y personal son, al final, los que dejan una huella indeleble en la vida personal y académica de su alumnado. En este sentido, cobra especial relevancia el papel de la vocación entendiéndola como una llamada y predisposición hacia el ejercicio educativo.

\title{
Hay maestros con una enorme vocación dispuestos a dar a los niños las herramientas para aprender a pensar. (S.150 - Prácticas III)
}

Este rasgo definitorio denota en los tutores una inclinación y compromiso hacia su labor docente, mostrando una actitud de entrega académica al educando en pos de su desarrollo integral (Álvarez et al., 2017), convirtiéndose en la categoría que más evidencias registró en el arquetipo del maestro auténtico y personal. Dicha pasión hacia la enseñanza se vehicula, por tanto, a través de su identidad y sus acciones (Day, 2014), de manera que educan más por lo que son que por lo que dicen o saben (Rassam, 1979). Así, su interioridad y riqueza personal se convierten en ejemplo y modelo a imitar por su alumnado y, también, por los estudiantes de magisterio en prácticas. Esta idea se refleja en la siguiente evidencia, donde el estudiante en Prácticas II muestra su admiración hacia el compromiso que su tutor del centro tiene respecto a la educación y su alumnado.

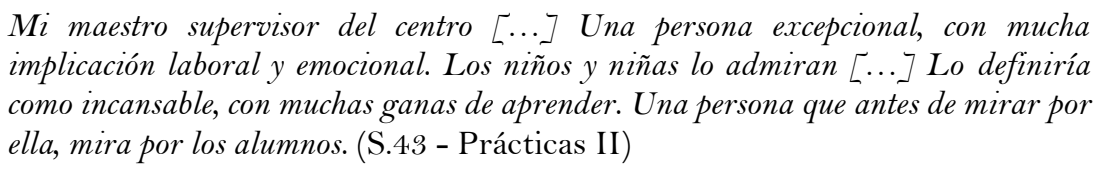

Por último, resaltar que los tutores de los centros también han mostrado preocupación por el desarrollo de su identidad profesional docente, dando relevancia a seguir aprendiendo y mejorando como profesionales para ejercer mejor su labor educativa con el alumnado (Bermúdez y Laspalas, 2017), una cuestión más valorada por el alumnado en prácticas IV que está finalizando el grado, al ser más conscientes de la relevancia de la formación continua para el crecimiento y desarrollo profesional.

Mi tutora hacía hincapié en que, como maestra, es importante conocerse a una misma como persona y descubrir nuevas fortalezas o debilidades para mejorarlas o subsanarlas. (S.203 - Prácticas IV) 


\subsubsection{Maestro transmisor de historias}

La tipología del maestro transmisor de historias también ha estado presente en los diarios de prácticas de los estudiantes de magisterio, aunque ha sido la que menor número de evidencias ha registrado. Centrándonos en las categorías establecidas en torno a los rasgos definitorios, los tutores que encarnan este arquetipo destacan por el dominio de los contenidos de su asignatura, siendo el factor clave la relación que son capaces de establecer entre su materia y los acontecimientos que ocurren en la vida real (Nussbaum, 2001). Sobre esta praxis reflexionan los futuros docentes, asumiendo la necesidad de proponer aprendizajes significativos que partan del interés y la realidad de los discentes. Este dominio de los contenidos y su aplicabilidad a contextos reales es valorado positivamente por ser un aspecto clave para poder desarrollar positivamente los procesos educativos.

Tener los conocimientos necesarios para poder impartir clases es primordial, pero me parece igual o más importante lograr interesar a los alumnos en la materia incorporando temas de actualidad o de su realidad, aspecto que mi tutora trabajaba cada día. (S.184 - Prácticas IV)

Me ha sorprendido especialmente su capacidad por mantener despierto el interés de los alumnos. (S.93 - Prácticas III)

He estado colaborando con un tutor el cual su objetivo era despertar la curiosidad del alumnado con respecto a los contenidos conectándolos, en la medida de lo posible, con los intereses del alumnado. (S.125 - Prácticas III)

Se trata de transformar las lecciones en experiencias didácticas que hagan vibrar al alumnado, como defiende Jordán y Codana (2019), logrando así cautivar al discente por encima de los contenidos que tengan que abordarse. Para conseguirlo, es clave el amor hacia la materia, logrando así maestros "abiertamente apasionados y claramente comprometidos con su docencia y sus alumnos” (Fried, 2001, p. 52). En los diarios se recogen evidencias que resaltan la existencia de tutores entusiasmados con el cuerpo de contenidos de su especialidad, donde las ganas por compartir los conocimientos de su área están por encima de todo esfuerzo pedagógico que deba realizar para lograr atrapar el deseo de aprender del alumnado. En este caso, es un estudiante de prácticas I quien analiza la forma en la que su tutor aborda el proceso educativo, convirtiéndose en un valioso ejemplo de cómo disfrutar el ejercicio docente en torno a su materia.

\footnotetext{
Le encanta su asignatura. Su método de enseñanza es participativo y motivador, expone el tema, lo adorna con anécdotas y constantemente hace preguntas a la clase para fomentar el diálogo y que ellos mismos formen su conocimiento movidos por la curiosidad. (S.20 - Prácticas I)
}

De esta forma, se refuerza la importancia que los contenidos y los conocimientos tienen en la formación de los futuros docentes, manteniendo su relevancia en la conformación de la identidad profesional docente.

\subsubsection{Maestro motivador para el aprendizaje}

Los tutores que responden al arquetipo de maestro motivador para el aprendizaje se caracterizan por abordar el hecho educativo dando prioridad a los elementos que determinan una adquisición eficiente y de calidad de los conocimientos, de la que deben asegurarse personalmente, empleando para ello todos los recursos didácticos y pedagógicos que estén a su alcance. La importancia de la competencia pedagógica, la segunda categoría con más evidencias registradas en este arquetipo, se refleja en el siguiente testimonio de un estudiante de prácticas IV, donde se da relevancia al saber didáctico como capacidad que se ha debido de adquirir a lo largo de la titulación. 
La metodología empleada por la maestra es altamente motivadora, con lo que se consigue enganchar a los niños para que sigan la clase con atención. (S.170 Prácticas IV)

En este sentido, la prioridad se centra en captar la atención y motivar al alumnado mediante procesos de aprendizaje innovadores y flexibles (Hargreaves, 2003), siendo el rasgo definitorio con mayor número de evidencias registradas. Para lograr dicha motivación, se puede hacer uso de diferentes metodologías (cuestión destacada por un estudiante de prácticas I) o mediante materiales para favorecer la experimentación y el interés (evidencia perteneciente a un alumno de prácticas IV), como se refleja en los testimonios recogidos a continuación:

\section{La didáctica que emplea la profesora es actual, orientando y guiando el aprendizaje de los niños de manera participativa, estando muy activos y motivados en clase. (S.5 - Prácticas I) \\ Los alumnos estaban teniendo el primer contacto con geometría, la tutora es muy buena y ha sabido captar su atención durante toda la clase, mediante la utilización de objetos similares a figuras geométricas. (S.137 - Prácticas III)}

Los estudiantes de magisterio pueden observar como en el aula se le da protagonismo a elementos más creativos y divertidos para afrontar los procesos de enseñanza-aprendizaje (Centeno et al., 2017), lo que les permite comprender de forma crítica los beneficios de esta praxis. Se trata de una reflexión más presente en estudiantes de prácticas II al no tratarse de su primera experiencia docente en prácticas, donde el interés se ha centrado en otro tipo de cuestiones.

\section{Mi tutora siempre ha intentado trabajar con actividades divertidas que fomentasen la responsabilidad, la creatividad, las habilidades comunicativas, etc. (S.48 - Prácticas II)}

Estamos, por tanto, ante docentes que son conscientes de la importancia de crear ambientes y contextos significativos para el aprendizaje, logrando así atraer, motivar y estimular a los discentes (Day, 2014), como recoge el siguiente testimonio.

Ella siempre me decía que lo importante del colegio era que los niños estuviesen contentos, motivados y con curiosidad por aprender cosas nuevas, si no se daba esa situación, el proceso de enseñanza-aprendizaje no sería fructífero. (S.62 Prácticas II)

Se convierten, por lo tanto, en ejemplos de calidad pedagógica y fomento de procesos de enseñanza-aprendizaje enriquecedores para el alumnado en prácticas.

\section{Conclusiones}

La investigación realizada ha permitido profundizar, desde la óptica del alumnado de magisterio durante sus periodos de prácticas, en el conocimiento de la figura del tutor del centro respecto a su forma de ser y ejercer como maestro. A través de las experiencias recopiladas en los diarios de prácticas, ha quedado manifestada la importancia de su labor y su ejemplo en la construcción de la identidad profesional docente, coincidiendo con la investigación de Egido y López (2016).

De esta manera, y tomando en consideración el objetivo general que perseguía este trabajo a partir de la propuesta de Esteban y Mellen (2016), el análisis pormenorizado de los diarios nos ha permitido identificar las tipologías de docentes que tutorizan a los futuros maestros durante su periodo de prácticas curriculares. En este sentido, debemos 
considerar que cada arquetipo de maestro está conformado por valores, actitudes, competencias, intereses y enfoques didácticos-pedagógicos diferentes. El hecho de conocer cómo son los tutores de los centros de prácticas y cómo afrontan los procesos de enseñanza-aprendizaje, desde la perspectiva de los propios estudiantes, nos permitirá identificar los diferentes arquetipos de maestros y los rasgos definitorios que representan a los tutores de prácticas. En lo que respecta al nivel de influencia de estas tipologías, estará determinado por dos realidades: la concepción previa sobre la figura docente que tengan los estudiantes de magisterio, a tenor de sus experiencias previas (Mellado et al., 2006); y la significatividad de las vivencias que experimenten con dichos arquetipos durante su estancia en el centro de prácticas.

Pasamos a exponer las principales conclusiones en torno a los objetivos específicos. Respecto al análisis de la frecuencia de los arquetipos y sus respectivos rasgos definitorios, es importante considerar que las características más frecuentes que manifiesten y desarrollen los tutores de los centros son los más fácilmente reproducibles por los estudiantes en prácticas, al convertirse en el primer ejemplo docente desde el inicio de los estudios de magisterio (Martínez y Raposo, 2011). De esta manera, la tipología del maestro auténtico y personal ha sido la que ha obtenido una mayor presencia en los diarios, coincidiendo con la investigación de Esteban y Mellen (2016). En este sentido, hay que destacar que en los diarios se recopilan mayor número de experiencias de corte emocional, coincidiendo con el estudio de López y otros (2015), siendo uno de los motivos por el cual predominan las evidencias ligadas a este arquetipo. Por su parte, el maestro motivador para el aprendizaje y el transmisor de historias han tenido menor presencia en los registros de los diarios, tal y como ocurría en el estudio de Esteban y Mellen (2016). Este hecho denota que, en la reflexión realizada por los futuros docentes sobre la experiencia formativa de prácticas, las características asociadas a dichos arquetipos han sido menos percibidas por los estudiantes o han sido menos valoradas de cara a su consideración para incluirlas en los diarios. Este hecho contradice la tesis de Ball, Thames y Phelps (2008), donde el dominio de los contenidos y de los recursos didácticos eran muy valorados por los futuros docentes.

Centrándonos en identificar cuáles son las diferencias en las percepciones de los estudiantes respecto a los arquetipos de tutores presentes en los centros en función del periodo de prácticas que cursen, hay que resaltar que la maduración profesional y el propio desarrollo formativo del alumnado (merced a la adquisición de competencias en el grado y a la realización de algún periodo de prácticas) influyen en su percepción sobre los rasgos definitorios y comportamientos del tutor del centro. En este sentido, los rasgos asociados al maestro auténtico y personal han registrado más evidencias en todos los periodos de prácticas que cualquier rasgo de las otras dos tipologías, destacando la vocación y el desarrollo profesional como un elemento clave para el ejercicio docente, coincidiendo con la tesis de Larrosa (2010) donde ambos aspectos deben conjugarse para una preparación profesional completa. Destacar como el arquetipo del maestro motivador para el aprendizaje ha aumentado el registro de sus evidencias en los últimos periodos de prácticas, donde el alumnado se encuentra finalizando sus estudios y posee un mayor nivel de competencia que en los primeros periodos de prácticas, dando mayor relevancia en dicha etapa a dominar las competencias pedagógicas necesarias para desarrollar procesos educativos motivadores y significativos, como defiende (González y Rodríguez, 2014). Por el contrario, los rasgos que definen al maestro transmisor de historias han tenido un bajo 
registro de evidencias, repartiéndose de forma equitativa si tenemos en cuenta el número de evidencias y de estudiantes en cada periodo de prácticas.

En cuanto al registro de evidencias sobre los rasgos definitorios de los tutores atribuidas a cada arquetipo a partir de la percepción de los estudiantes, como se ha expuesto en la fundamentación teórica, los comportamientos y actitudes que los tutores exterioricen al abordar el proceso de enseñanza-aprendizaje serán los que más influyan en la conformación de la identidad docente del alumnado en prácticas. De este modo, los tutores identificados como maestros auténticos y personales se caracterizan por priorizar sobre el factor humano y por cuidar las relaciones profesionales y personales con su alumnado mediante la regulación y gestión emocional, obteniendo las subcategorías competencia emocional y desarrollo y bienestar personal de los estudiantes un total de 119 evidencias registradas $(56,1 \%)$, siendo la suma de ambas mayoría dentro de dicho arquetipo, correspondiéndose con la tesis de Day (2011). Por su parte, los tutores que se asocian al rol de transmisor de historias destacan por la pasión y el dominio y aplicabilidad de su materia, centrando su esfuerzo en conseguir despertar el deseo de aprender del discente, en consonancia con el estudio de Jordán y Codana (2019). No obstante, ha sido el arquetipo con el menor número de evidencias registradas, siendo menos relevantes estos rasgos que los asociados a otros arquetipos con un registro mayor. En cuanto a los tutores que se vinculan a la tipología del maestro motivador para el aprendizaje, los rasgos definitorios más destacados son la motivación y la creatividad (39 evidencias, suponiendo un 66,1\% del total del arquetipo), relacionándose con la capacidad para desarrollar procesos educativos innovadores y creativos que logren implicar al alumnado, coincidiendo con los estudios de Centeno y otros (2017), Day (2014) y Hargreaves (2003). Un aspecto que ha ido aumentando conforme avanzaban los periodos de prácticas, dando más relevancia al saber pedagógico en las etapas finales del grado.

Como limitación, destacar que la muestra de estudio describió sus experiencias y vivencias de forma libre, sin ningún tipo de pautas, lo que ha dificultado la realización de una reflexión más profunda por parte de los estudiantes sobre el papel del tutor del centro. Este trabajo de estructuración de las evidencias registradas en los diarios ha recaído en los investigadores, teniendo que asociar las diferentes experiencias a las categorías establecidas para cada arquetipo (rasgos definitorios). Entre las líneas futuras de investigación, se situará el foco en profundizar sobre la figura del tutor del centro. Se podría realizar, por ejemplo, una comparativa entre los arquetipos de los tutores presentes en diferentes etapas educativas (infantil, primaria y secundaria), desarrollando procesos de autorreflexión sobre la IPD de los tutores y definiendo requisitos de carácter competencial, actitudinal y comportamental para ejercer la labor de tutorización de futuros maestros.

\section{Referencias}

Aguaded, M. C. y Pantoja, M. J. (2015). Innovar desde un proyecto educativo de inteligencia emocional en primaria e infantil. Tendencias Pedagógicas, 26, 69-88.

Aguilar, C. (2017). La tertulia pedagógica dialógica en el practicum de la formación inicial de maestras y maestros. Revista Iberoamericana de Educación, 73(2), 9-22.

Álvarez, J. L., Martínez, M. J., González, H. y Buenestado, M. (2017). El aprendizaje-servicio en la formación del profesorado de las universidades españolas. Revista Española de Pedagogía, 75(267), 199-2 17. https://doi.org/10.22550/REP75-2-2017-02 
Applegate, J. R. (2010). Teaching competencies and the teacher preparation program. Improving College and University Teaching, 25(4), 226-230.

https://doi.org/10.1080/00193089.1977.9927488

Ball, D. L., Thames, M. H. y Phelps, G. (2008). Content knowledge for teaching: What makes it special? Journal of Teacher Education, 59, 389-394. https://doi.org/10.1177/0022487108324554.

Beauchamp, C. y Thomas, L. (2009). Understanding teacher identity: An overview of issues in the literature and implications for teacher education. Cambridge Journal of Education, 39(2), 175189. https://doi.org/10.1080/03057640902902252

Bermúdez, J. J. y Laspalas, F. J. (2017). El profesor universitario: Integración entre lo personal y lo profesional. Teoría de la Educación. Revista interuniversitaria, 29(2), 109-126. https://doi.org/10.14201/teoredu292109126

Bodas, E., González, R. y Martín, A. M. (2016). La adquisición de las competencias en el practicum II del máster de formación del profesorado a través de las actividades de aprendizaje y de la interacción en la tutoría. En J. Bernal (Coord.), Actas del XIV Congreso Interuniversitario de Organización de Instituciones Educativas (pp. 417-421). Zaragoza: Universidad de Zaragoza.

Buxarrais, M. R., Vilafranca, I. y Bujons, C. (2016). Los contenidos en la formación de maestros y su incidencia en el carácter. En F. Esteban (Ed.), La formación del carácter de los maestros (pp. 73-88). Barcelona: Ediciones de la Universitat de Barcelona.

Cabero, J., García, L., Query del Moral, P. y García de la Concha, D. (2016). La televisión universitaria por internet: Análisis de la situación española. Edutec. Revista Electrónica de Tecnología Educativa, 55, 1-16. https://doi.org/10.21556/edutec.2016.55.578

Cantón, I. y Tardif, M. (Coords.). (2018). Identidad profesional docente. Madrid: Narcea.

Cebrián, D., Pérez, R. y Cebrián, M. (2017). Estudio de la comunicación en la evaluación de los diarios de prácticas que favorecen la argumentación. Revista Prácticum, 2(1), 1-21.

Centeno, G., Alcaraz, A. L., González, O. R., Oropeza, K. S. y Ramírez, G. (2017). El lenguaje, una herramienta para aprender mediante el modelo de gestión aprendizaje invertido. Un estudio de caso en alumnos de $5^{\circ}$ de primaria. Edutec. Revista Electrónica de Tecnología Educativa, 59, 1-17. https://doi.org/10.21556/edutec.2017.59.756

Cid, A., Pérez, A. y Sarmiento, J. A. (2011). La tutoría en el practicum. Revisión de la literatura. Revista de Educación, 354, 127-154.

Colén, M. T. y Castro, L. (2017). El desarrollo de la relación teoría y práctica en el grado de maestro en educación primaria. Profesorado. Revista de Curriculum y Formación del Profesorado, 21(1), 59-79.

Colomo, E. y Domínguez, R. (2015). Definiendo identidades: El canciograma como herramienta metodológica de autoconocimiento. REICE. Revista Iberoamericana sobre Calidad, Eficacia y Cambio en Educación, 13(2), 131-146.

Colomo, E. y Oña, J. M. (2014). Pedagogía de la muerte. Las canciones como recurso didáctico. REICE. Revista Iberoamericana sobre Calidad, Eficacia y Cambio en Educación, 12(3), 109-121.

Corral, M. J. y Cacheiro, M. L. (2016). Los recursos TIC y el eportfolio como estrategia para la interacción didáctica en secundaria: Estudio de caso. Revista de Humanidades, 28, 115-138. https://doi.org/10.5944/rdh.28.2016.16496

Day, C. (2009). A passion for quality: Teachers who make a difference. Tijdschrift Voor Lerarenopleiders, 30(3), 4-13. 
Day, C. (2011). Uncertain professional identities: Managing the emotional contexts of teaching. En C. Day y J. Chi-Kin Lee (Eds.), New understandings of teacher's work: Emotions and educational change (pp. 45-64). Dordrecht: Springer. https://doi.org/10.1007/978-94-007-0545-6_4

Day, C. (2014). Pasión por enseñar. Madrid: Narcea.

Day, C. (2017). Teacher's worlds and word. Londres: Routledge. https://doi.org/10.4324/9781315170091

Egido, I. y López, E. (2016). Condicionantes de la conexión entre la teoría y la práctica en el prácticum de magisterio. Algunas evidencias a partir de TEDS-M. Estudios Sobre Educación, 30, 217-237. https://doi.org/10.15581/004.30.217-237

Esteban, F. (2018). Ética del profesorado. Barcelona: Herder.

Esteban, F. y Mellen, T. (2016). ¿Por qué quieres ser maestro? ¿Cómo es un buen maestro? Ideas para la formación universitaria. Bordón, 68(2), 185-198.

https://doi.org/10.13042/Bordon.2016.68212

Esteve, J. M. (2009). La formación de profesores: Bases teóricas para el desarrollo de programas de formación inicial. Revista de Educación, 350, 15-29.

Fontal, O., Ibáñez, A., Martínez, M. y Rivero, P. (2017). El patrimonio como contenido en la etapa de primaria, del currículum a la formación de maestros. Revista Electrónica Interuniversitaria de Formación del Profesorado, 20(2), 79-95. https://doi.org/10.6018/reifop/20.2.28632 1

Frankl, V. (2013). El hombre en busca del sentido. Barcelona: Herder.

Fried, R. L. (2001). The passionate teacher. A practical guide. Boston, MA: Beacon.

García, E. (2017). El portafolio como metodología de enseñanza-aprendizaje y evaluación en el practicum: Percepciones de los estudiantes. REDU. Revista de Docencia Universitaria, 15(1), 241-257. https://doi.org/10.4995/redu.2017.6043

García, S. M., González, R. y Martín, A. M. (2016). Influencia de las prácticas en el desarrollo de la identidad profesional de los estudiantes de educación social. Revista Interuniversitaria de Pedagogía Social, 28, 245-259. https://doi.org/10.7179/PSRI_2016.28.18

González, M. (2015). El practicum en la formación del profesorado de secundaria. Revista Española de Pedagogía, 261, 301-319.

González, M. y Fuentes, E. J. (2011). El practicum en el aprendizaje de la profesión docente. Revista de Educación, 354, 47-70.

González, M. y Rodríguez, B. (2014). La formación inicial de los profesores de lengua extranjera: Un espacio para generar estilos de actuación. Bordón, 66(4), 69-86. https://doi.org/10.13042/Bordon.2014.66406

González, R., Martín, A. M. y Bodas, E. (2017). Adquisición y desarrollo de competencias docentes en el practicum del máster de secundaria: Las actividades de aprendizaje y la tutoría. Revista de Humanidades, 31, 153-174. https://doi.org/10.5944/rdh.31.2017.19077

González, R. y Medina, A. (2017). El desarrollo profesional de los docentes de educación infantil. En A. Medina, A. de la Herrán y M. C. Domínguez (Coords.). Formación de profesorado de frontera (pp. 23-58). Madrid: UNED.

Granados, J., Tapia, A. M. y Fernández, J. (2017). La construcción de la identidad de los docentes noveles: Un análisis desde las teorías apriorísticas. REDU. Revista de Docencia Universitaria, 15(2), 163-178. https://doi.org/10.4995/redu.2017.6746 
Hargreaves, A. (2003). Teaching in the knowledge society: Education in the age of insecurity open. Philadelphia, PA: University Press. https://doi.org/10.1016/j.tate.2005.06.007

Hargreaves A. (2005). Educational change takes ages: Life, career and generational factors in teachers' emotional responses to educational change. Teaching and Teacher Education, 21(8), 967-983.

Hong, J., Greene, B. y Lowery, J. (2017). Multiple dimensions of teacher identity development from pre-service to early years of teaching: A longitudinal study. Journal of Education for Teaching. International research and pedagogy, 43(1), 1-15. https://doi.org/10.1080/02607476.2017.1251111

Jordán, J. A. (2003). Influencia tácita del profesor y educación moral informal. Revista Española de Pedagogía, 61(224), 153-172.

Jordán, J. A. (2013). Pertinencia social y compromiso ético en la tarea docente. En M. Esteban Villar (Coord.), El prestigio de la profesión docente en España. Percepción y realidad (pp.122124). Madrid: Fundación Botín y Fundación Europea Sociedad y Educación.

Jordán, J. A. y Codana, A. (2019). La influencia del profesor apasionado en la mejora académica y el desarrollo personal de sus alumnos. Estudios Sobre Educación, 36, 31-51. https://doi.org/10.15581/004.36.31-51

Jung, C. (1992). Psicología y simbólica del arquetipo. Barcelona: Paidós.

Jung, C. (1994). Arquetipos e inconsciente colectivo. Barcelona: Paidós.

Larrosa, F. (2010). Vocación docente versus profesión docente en las organizaciones educativas. Revista Electrónica Interuniversitaria de Formación del Profesorado, 13(4), 43-51.

Leguizamon, G. (2014). La construcción de saberes pedagógicos en la formación del profesorado. REICE. Revista Iberoamericana sobre Calidad, Eficacia y Cambio en Educación, 12(1), 35-54.

Lopes, H. A. y Blázquez, F. (2012). La práctica pedagógica en la formación inicial de profesores del primer ciclo de enseñanza básica en Portugal. Enseñanza E゚ teaching. Revista Interuniversitaria de Didáctica, 30(1), 23-43.

López, E. (2016). La formación docente del profesorado universitario: Sentido, contenido y modalidades. Bordón, 68(4), 89-102. https://doi.org/10.13042/Bordon.2016.38998

López, M. A., Torrego, L. M. y Vallés, C. (2015). ¿Qué metáforas personales definen al profesorado de educación infantil en formación? REICE. Revista Iberoamericana sobre Calidad, Eficacia y Cambio en Educación, 13(3), 37-56.

Manso, J. y Martín, E. (2014). Valoración del máster de formación de profesorado de educación secundaria: Estudio de casos en dos universidades. Revista de Educación, 364, 145-169.

Martín, A., Conde, J. y Mayor, C. (2014). La identidad profesional docente del profesorado novel universitario. REDU. Revista de Docencia Universitaria, 12(4), 141-160. https://doi.org/10.4995/redu.2014.5618

Martínez, E. y Raposo, M. (2011). Funciones generales de la tutoría en el practicum: Entre la realidad y el deseo en el desempeño de la acción tutorial. Revista de Educación, 354, 155-181.

Matsuba, M. K., Murzyn, T. y Hart, D. (2011). A model of moral identity: Applications for education. Advances in Child Development and Behavior, 40, 181-207. https://doi.org/10.1016/B978-0-12-386491-8.00005-0

Medina, A., Holgueras, A. I., Martín-Cuadrado, A. M., González, R. y Campos, B. (2013). Análisis de la formación práctica. Base de la iniciación profesional del profesorado de educación secundaria. En P. C. Muñoz., M. Raposo, M. González, M. E. Martínez, M. Zabalza y A. 
Pérez (Coords.), Un practicum para la formación integral de los estudiantes (pp. 1219-1228). Santiago de Compostela: Andavira.

Melgarejo, J., Pantoja, A. y Latorre, P. A. (2014). Análisis de la calidad del practicum en los estudios de magisterio desde la perspectiva del alumnado. Aula de Encuentro, 16(1), 53-70.

Mellado, V., Ruiz, C., Bermejo, M. L. y Jiménez, R. (2006). Contributions from the philosophy of science to the education of science teachers. Science and Education, 15(5), 419-445. https://doi.org/10.1007/s1 1191-005-8920-y

Mojarro, A., Rodrigo, D. y Etchegaray, M. C. (2015). Educación personalizada a través de elearning. Alteridad, 1O(1), 21-30. https://doi.org/10.17163/alt.v10n1.2015.02

Muñoz, M. (2010). Educar desde la compasión apasionada. REICE. Revista Iberoamericana sobre Calidad, Eficacia y Cambio en Educación, 8(2), 217-223.

Nussbaum, M. (2001). El cultivo de la humanidad. Barcelona: Editorial Andrés Bello.

Oakeshott, M. (2009). La voz del aprendizaje liberal. Buenos Aires: Katz Editores.

Ordine, N. (2013). La utilidad de lo inútil. Manifiesto. Barcelona: Acantilado.

Palomares, A. y Alarcón, M. C. (2018). El tutor de prácticas y su influencia en la formación de profesionales en los Grados de Educación. Revista Prácticum, 3(1), 34-47.

Pennac, D. (2008). Mal de escuela. Barcelona: Mondadori.

Quevedo, E. y Arruti, M. A. (2018). El nuevo rol del docente como facilitador del aprendizaje. En A. Villa (Ed.), Tendencias actuales de las transformaciones de las universidades en una nueva sociedad digital (pp. 195-208). Bilbao: Foro Internacional de Innovación Universitaria.

Ralph, E. G. y Walker, K. D. (2014). Mentorship in the practicum: Post-interns' perspectives. International Journal of Humanities and Social Science, 4(8), 1-10.

Ramírez, M. S. y De la Herrán, A. (2012). La madurez personal en el desarrollo profesional del docente. REICE. Revista Iberoamericana sobre Calidad, Eficacia y Cambio en Educación, 1O(3), 25-44.

Rassam, J. (1979). Le professeur et les élèves. Revue Thomiste, 76(1), 59-76.

Recalcati, M. (2016). La hora de clase. Por una erótica de la enseñanza. Barcelona: Anagrama.

Ries, F., Yanes, C. y Ballesteros, M. A. (2014). The training needs of future secondary education teachers in Spain. International Journal for Crossdisciplinary Subjects in Education, 5(2), 16611668. https://doi.org/10.20533/ijcdse.2042.6364.2014.0233

Romera, M. J. (2011). La investigación-acción en la formación del profesorado. Revista Española de Documentación Científica, 34, 597-614. https://doi.org/10.3989/redc.2011.4.836

Santamaría, N., Torrego, L. y Ortiz, R. (2018). Identidad profesional docente y movimientos de renovación pedagógica. Tendencias Pedagógicas, 32, 177-192. https://doi.org/10.15366/tp2018.32.013

Sarceda, C. (2017). La construcción de la identidad docente en la educación infantil. Tendencias Pedagógicas, 30, 281-300.

Sauquillo, P., Ros, C. y Bellver, M. C. (2008). El rol de género en los videojuegos. Teoría de la Educación. Educación y Cultura en la Sociedad de la Información, 9(3), 130-149.

Scott, G. A. (2000). Plato’s Socrates as educator. Nueva York, NY: SUNY Press.

Sepúlveda, M. P., Gallardo, M., Mayorga, M. J. y Madrid, D. (2017). La evaluación del practicum: Un proceso clave en la construcción y reconstrucción del pensamiento práctico. ENSAYOS. Revista de la Facultad de Educación de Albacete, 32(1), 93-110. 
Steiner, G. y Ladjali, C. (2005). Elogio de la transmisión. Madrid: Siruela.

Tejada, J., Carvalho, M. L. y Ruiz, C. (2017). El practicum en la formación de maestros: Percepciones de los protagonistas. Magis. Revista Internacional de Investigación en Educación, 9(19), 91-114. https://doi.org/10.11144/Javeriana.m9-19.pfmp

Tejada, J. y Ruiz, C. (2013). Significación del practicum en la adquisición de competencias profesionales que permiten la transferencia de conocimiento a ámbitos propios de la acción docente. Profesorado. Revista de currículum y formación del profesorado, 17, 91-110.

Toterhi, J. y Hancock, D. R. (2007). Exploring the relationship between certification sources, experience levels, and classroom management orientations of classroom teachers. Teaching and Teacher Education, 23(7), 1206-1216. https://doi.org/10.1016/j.tate.2006.04.013

Travé, G., Estepa, J. y Delval, J. (2017). Análisis de la fundamentación didáctica de los libros de texto de conocimiento del medio social y cultural. Educación XXI. Revista de la Facultad de Educación, 20(1), 319-338. https://doi.org/10.5944/educxx 1.17514

Valle, J. M. y Manso, J. (2018). El practicum en la formación inicial: Aportaciones del modelo 9:20 de competencias docentes. Cuadernos de Pedagogía, 489, 33-40.

Zabalza, M. A. (2011). El practicum en la formación universitaria: estado de la cuestión. Revista de Educación, 354, 21-43.

Zabalza, M. A. (2016). El practicum y las prácticas externas en la formación universitaria. Revista Practicum, 1(1), 1-23.

\section{Breve CV de los autores}

\section{Ernesto Colomo}

Doctor en Ciencias de la Educación por la Universidad de Málaga. Licenciado en Pedagogía, Diplomado en Magisterio con la especialidad de Educación Física, y Máster en Cambio Social y Profesiones Educativas por la misma universidad. Profesor Adjunto del Área de Educación en la Universidad Internacional de Valencia. Sus principales líneas de investigación se centran en la axiología educativa, la formación del profesorado, la pedagogía de la muerte y el análisis de elementos culturales para la educación e innovación educativa. Miembro de la Sociedad Española de Pedagogía, pertenece a varios comités científicos y es revisor de diferentes revistas científicas del ámbito pedagógico. ORCID ID: https://orcid.org/oOOO-OOO2-3527-7937. Email: ecolomomagana@gmail.com

\section{Vicente Gabarda}

Doctor en Pedagogía por la Universidad de Valencia. Profesor Adjunto del Área de Educación en la Universidad Internacional de Valencia. Experiencia en docencia y gestión universitaria, dirigiendo programas y departamentos e implementando proyectos de innovación docente en entornos presenciales y virtuales. Autor de publicaciones en revistas indexadas de impacto, co-autor de capítulos de libro y publicaciones/contribuciones en Congresos Nacionales e Internacionales y evaluador de artículos de investigación en revistas indexadas. Entre sus principales líneas de investigación destacan los procesos de enseñanza y aprendizaje en contextos e-learning, la formación del profesorado y la innovación educativa. ORCID ID: https://orcid.org/oOOO-OOO1-6159-5173. Email:vgabarda@gmail.com 